\title{
Mathematical Modelling and Computer Simulation of the Flow in Thin Gap Channel due to Alternating Volumetric Mass Forces
}

\author{
IVAN V. KAZACHKOV ${ }^{1,2}$ \\ ${ }^{1}$ Department of Information Technology and Data Analysis \\ Nizhyn Mykola Gogol State University, 16600 Grafska str. 2 Nizhyn, UKRAINE, \\ ${ }^{2}$ Department of Energy Technology \\ Royal Institute of Technology, 10044 Brinellvägen 68 Stockholm, SWEDEN,
}

Abstract: - The mathematical modelling and computer simulation are presented for the complex flow in thin gap channel due to alternating volumetrically distributed mass forces. The flow equations and obtained analytical solutions for limit cases are considered in the cylindrical coordinate system with the axis directed along the channel, which is rotating around its axis. The channel is placed inside the cylinder on the edge of the circular horizontal disk, which is rotating around vertical axis in its centre. The two rotations around different perpendicular axes create complex unknown features in a flow due to the alternating centrifugal and Coriolis forces, which substantially vary by the angle. The centrifugal force from the disk rotation is directed to its edge, while the centrifugal force due to rotation of the channel is acting by the channel's radius. As a result, the two different centrifugal forces are directed counter currently in one side of the channel and vary by the angle up to adding of the two of them in the same direction in the opposite side of the channel. The conditions may fit to the strong cavitation regime inside the volume of fluid flow due to a stretching of the liquid in some locations.

Key-Words: - Alternating Mass Forces; Vertical and Horizontal Axes; Cylindrical Gap; Centrifugal and Coriolis Forces; Stretching of Liquid

Received: April 30, 2020. Revised: November 25, 2020. Accepted: December 2, 2020. Published: December 14, 2020.

\section{Introduction and the Problem Statement}

\subsection{Description of the System}

The complex flow in the rotational channel placed on the rotating horizontal disk is considered according to the schematic shown in Fig. 1:

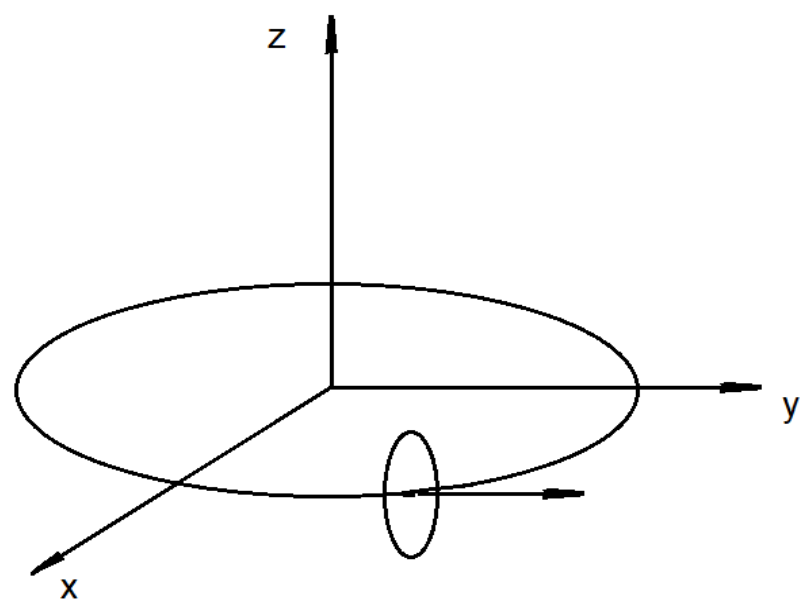

Fig. 1. Schematic of the flow in channel placed on the disk rotating around vertical axis
Horizontal disk is rotating around the vertical axis and the gap flow is considered in the channel placed on this disk. The channel rotates inside the cylinder around the tangential axis to the edge of disk with the radius $\mathrm{R}_{0}$. The centrifugal forces are shown in Fig. 2:

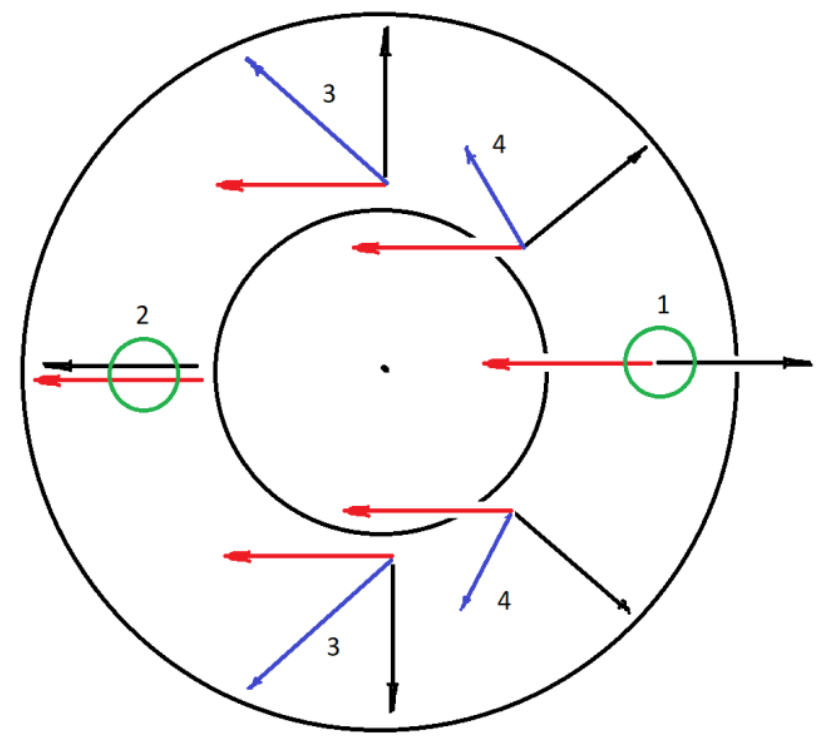

Fig. 2 Centrifugal forces in flow due to rotation of channel and rotation of disk on horizontal plate 
Thus, in the situation 1 in Fig. 2, the forces act counter currently causing a high stretching in a liquid (condition for the cavitation process). Centrifugal forces due to the disk's rotation (red colour) are directed in all points of the flow to the left in picture (edge of the disk), while centrifugal forces due to rotation of the cylindrical channel (black in colour) act by the radius of the channel.

The rotation of the horizontal disk is going around the vertical axis with the speed $\Omega$. The other rotation is done with the speed $\omega$ regarding the tangential axis to the circle of main rotation of the horizontal disk. The internally rotating channel with the radius $r_{0}$ is located on the disk inside the cylinder oriented along the tangential line (see Fig. 1), at the distance $R_{0}$ from the central vertical axis $z(x=y=0)$ of the horizontal disk.

The liquid is pumped into the rotating channel. For example, by rotation with the speed of 3000 , 6000, 12000 and $18000 \mathrm{rpm}$ (rpm=1/60 1/s), respectively. It results in the next estimations for the flow velocity and for the pressure at the inlet:

Table 1 Parameters of rotation and flow in channel

\begin{tabular}{|l|c|c|c|c|}
\hline $\begin{array}{l}\Omega, \\
1 \mathrm{rps}= \\
60 \mathrm{rpm}\end{array}$ & $\begin{array}{l}3000 \\
\mathrm{rpm}= \\
501 / \mathrm{s}\end{array}$ & $\begin{array}{l}6000 \\
\mathrm{rpm}= \\
1001 / \mathrm{s}\end{array}$ & $\begin{array}{l}12000 \\
\mathrm{rpm}= \\
2001 / \mathrm{s}\end{array}$ & $\begin{array}{l}18000 \\
\mathrm{rpm}= \\
3001 / \mathrm{s}\end{array}$ \\
\hline $\mathrm{V}, \mathrm{m} / \mathrm{s}$ & 15 & 30 & 60 & 90 \\
\hline$\Delta p_{\mathrm{Bar}}$ & 1.1 & 4.5 & 18 & 38 \\
\hline $\mathrm{N}, \mathrm{kW}$ & 0.113 & 0.452 & 1.81 & 4.07 \\
\hline
\end{tabular}

The last row of the Table 1 shows the power for corresponding rotation by the mass flow rate $1 \mathrm{~kg} / \mathrm{s}$. Here it is $1 \mathrm{rps}=1 / \mathrm{s}$. Let us consider water flow going in a gap between the two rotating cylindrical channels. If accept that the pressure difference is just a half of the corresponding $\Delta p$, then one can get for the square $2.52 * 10^{-6} \mathrm{~m}^{2}$ with this situation, the values of the force as follows: $3.1,12.4$ and $49.6 \mathrm{~N}$, respectively, for $\Omega=6000,12000,18000 \mathrm{rpm}$.

Neglecting the energy loses, with the 100 tilted 45 degree holes between the internal channels, we get the rotation force 310,1240 and $2790 \mathrm{~N}$. This creates approximately the same rotation speed as the main one: $\omega=6600,13200$ and 19800 , respectively.

\subsection{Cylindrical Coordinates in Channel}

The rotating coordinate system can be done with the vertical axis coinciding with $z$ or shifted from the central axis $(x=y=0)$ on some distance $R_{0}$ as shown in Fig. 1. The rotation is going around the vertical axis $z$ and also around the axis tangential to the circle of the radius $\mathrm{R}_{0}$.

Intensive rotation and mixing flow are fascinating phenomena and may be highly effective in a number of applications: engineering, technological, natural processes [1-5]. Many theoretical aspects have been studied for the diverse rotational flows. Nevertheless, it is still the problem to dig more in deep for many theoretical, as well as practical applications. The described system was not considered yet.

In the local cylindrical coordinate system $(r, \varphi, z)$ connected to the channel, the coordinate surfaces are cylinders $r=$ const , semi-planes $\varphi=$ const and planes $z=$ const . Coordinate $\mathrm{z}$ is now directed by the axis of the cylinder because the paper is focused on the flow regimes in the gap of two rotating channels.

\subsection{Negative Pressure and Cavitation due to the Volumetric Stretching of Liquid}

In the above analysis it is seen that the liquid may get into stretching conditions in some local points, where the unknown dramatic regimes with negative pressure and cavitation are available. Normally cavitation is supposed to be under depressurization below the saturation level, due to which the liquid starts vaporizing in such local region. As concern to negative pressure corresponding to the local stretching of liquid that can also break the bonds of liquid molecules causing the cavitation process, it is much less known.

A stretched liquid is a liquid under negative pressure. This is an unstable, metastable state of a liquid, possible due to the Van der Waals forces of attraction between the molecules of the liquid - both between themselves and between them and the walls of the vessel. Real gas - in the general case - the gaseous state of a really existing substance [6], a gas that is not described exactly by the Clapeyron Mendeleev equation, in contrast to its simplified model - a hypothetical ideal gas strictly obeying the such equation.

Normally, a real gas is understood as a gaseous state of a substance in the entire range of its existence but there is another classification, according to which a highly superheated vapour is called a real gas, the state of which slightly differs from the state of an ideal gas, and superheated vapour, the state of which differs significantly from an ideal gas, and saturated vapour (two-phase equilibrium system liquid vapour), which does not obey the laws of an ideal gas at all [6]. This phenomenon can be observed in the Torricelli experiment. When lifting a tube filled with 
mercury, sealed from one end of the tube, the Torricelli void does not appear immediately. And with careful lifting of the tube, it is possible to achieve that the top of the tube completely filled with mercury is higher than the level corresponding to the current atmospheric pressure.

Similarly, mercury in the medical thermometer, after the contact with the body has ceased, is in a stretched state. And also it is in the maximum thermometer when the temperature begins dropping after the maximum [7].

Water can also be stretched, but this is only possible if it is thoroughly cleaned and degassed. In experiments with such water, the short-term tensile stresses of 23-28 MPa were achieved [8]. Technically pure liquids containing suspended solids and the smallest gas bubbles cannot withstand even minor tensile stresses. Nevertheless, this is a method of raising liquid that is used in trees [9].

Superheated (metastable) liquid heated above its boiling point causes such specific dynamic phenomena as explosive boiling due to a stored heat, instability of the liquid-vapour interface, and the formation of a front of phase transition in a number of the energy and technological regimes [10]. Water belongs to a class of substances that presents density anomalies [11]. These conditions may cause the different unique phenomena, e.g. cavitation and abnormal fluid (e.g. water) behaviours.

The negative pressure despite long history of study is still very little known phenomena [10-26], e.g. paper [10] shows using a few approaches that a high average stress difference on the interface of phase change is due to the negative stresses in the interface because the water belongs to a class of substances with density anomalies.

The negative pressure region of phase diagram proves to be paramount in understanding the unusual behaviour of this class of substances. It was demonstrated using theoretical arguments and experimental evidence that any condensed (solid or liquid) phase can exist in absolute negative pressure regimes, while the same is not true for gas phases. While in a gas phase pressure and density are proportional, this proportionality does not necessarily occur in condensed phases.

It is convenient to extend definition of pressure. In liquids and solids, pressure ought to be treated as $3 \times 3$ - tensor $\mathbf{P}$, rather than scalar [13]. The authors [11] have shown how the negative pressure region of the phase diagram proves to be paramount in understanding the unusual behaviour of this class of substances and in liquids and solids.

Huygens, Boyle, Papen, etc. made several experiments during a Royal Society Meeting [14] but they did not provide any explanation to the experiment because adhesion and cohesion where not known for them yet. To generate a very high negative pressure in a liquid one ought to use extremely small amounts of sample [15-24]. Several different methods were developed to generate negative pressures in liquids and a few hundreds of MPa were achieved. For example, the dynamic methods produce negative pressures for a very short period of time using the sudden pulling or ultrasonic radiation.

The study of boiling superheated and stretched liquids has been performed in a series of papers [2731]. The suppression effect for cavitation centres of a heterogeneous nature with the low-boiling impurities was discovered experimentally. The results on suppression of heterogeneous cavitation centres present practical possibilities for control of the boiling centres in a superheated stretched liquid. The effect of a gas dissolved in a liquid on the cavitation strength of liquids and the possibility of suppressing the centres cavitation by gas dissolved in liquid and other low-boiling impurities were studied as well.

The effect of a pulsed electric field on the limiting overheating of liquid at negative pressures was revealed. It was shown that for the short-term exposure to an electric field that does not lead to the formation of a noticeable amount of electrolysis products, the tension field $10^{7} \mathrm{~V} / \mathrm{cm}$ is not enough to change the temperature of the limit liquid overheating. The growth rate of vapour bubbles in the superheated stretched liquid was experimentally measured for the first time. Analysis of the factors determining the growth rate of vapour bubbles is very interesting. It was shown that a steady state of steam is not reached in a growing bubble in a stretched superheated liquid because it has not enough time for this process.

The physical situation described in the present paper has revealed negative pressure oscillations due to a strong volumetric variation of the mass forces in a space by the level and direction. The new features as oscillations of the flow parameters and pressure oscillations from the high positive to the high negative values in liquid were revealed in our numerical simulation as shown in the paper.

\section{Mathematical Model Development}

\subsection{Flow Equations Accounting Volumetric Alternating Mass Forces}

The Navier-Stokes equation array for the flow in the gap between rotating cylindrical channels, with 
account of the two centrifugal forces and the Coriolis force are written as follows

$$
\begin{aligned}
& \frac{\partial \rho}{\partial t}+\frac{\partial(\rho u)}{\partial r}+\frac{\rho u}{r}+\frac{\partial(\rho v)}{r \partial \varphi}+\frac{\partial(\rho w)}{\partial z}=0, \\
& \frac{\partial u}{\partial t}+u \frac{\partial u}{\partial r}+\frac{v \partial u}{r \partial \varphi}+w \frac{\partial u}{\partial z}-\frac{v^{2}}{r}+2(v \omega-w \Omega \cos \varphi)+ \\
& +\left(r_{0} \cos \varphi-R_{0}\right) \Omega^{2} \cos \varphi+r_{0} \omega^{2}=-\frac{1}{\rho} \frac{\partial p}{\partial r}+ \\
& +\frac{\partial}{r^{2} \partial \varphi}\left(v \frac{\partial u}{\partial \varphi}\right)+\frac{\partial}{\partial r}\left(v \frac{\partial u}{\partial r}\right)+\frac{\partial}{\partial z}\left(v \frac{\partial u}{\partial z}\right)+ \\
& +\frac{\partial(v u)}{r \partial r}-\frac{v}{r^{2}}\left(2 \frac{\partial v}{\partial \varphi}+u\right) \\
& \frac{\partial v}{\partial t}+u \frac{\partial v}{\partial r}+\frac{v \partial v}{r \partial \varphi}+\frac{w \partial v}{\partial z}+\frac{u v}{r}+2(w \Omega \sin \varphi-u \omega)+ \\
& +\left(R_{0}-r_{0} \cos \varphi\right) \Omega^{2} \sin \varphi=-\frac{1}{\rho r} \frac{\partial p}{\partial \varphi}+\frac{\partial}{\partial r}\left(v \frac{\partial v}{\partial r}\right)+ \\
& +\frac{\partial}{r^{2} \partial \varphi}\left(v \frac{\partial v}{\partial \varphi}\right)+\frac{\partial}{\partial z}\left(v \frac{\partial v}{\partial z}\right)+\frac{\partial(v v)}{r \partial r}+\frac{v}{r^{2}}\left(2 \frac{\partial u}{\partial \varphi}-v\right), \\
& \frac{\partial w}{\partial t}+u \frac{\partial w}{\partial r}+\frac{v \partial w}{r \partial \varphi}+w \frac{\partial w}{\partial z}+2 \Omega(v \sin \varphi-u \cos \varphi) \\
& =-\frac{1}{\rho} \frac{\partial p}{\partial z}+\frac{\partial}{\partial r}\left(v \frac{\partial w}{\partial r}\right)+\frac{\partial}{r^{2} \partial \varphi}\left(v \frac{\partial w}{\partial \varphi}\right)+ \\
& +\frac{\partial}{\partial z}\left(v \frac{\partial w}{\partial z}\right)+\frac{\partial(v w)}{r \partial r}, \\
& \rho c_{V}\left(\frac{\partial T}{\partial t}+u \frac{\partial T}{\partial r}+\frac{v \partial T}{r \partial \varphi}+\frac{w \partial T}{\partial z}\right)+ \\
& +p\left(\frac{\partial u}{\partial r}+\frac{u}{r}+\frac{\partial v}{r \partial \varphi}+\frac{\partial w}{\partial z}\right)=\frac{\partial}{\partial r}\left(\kappa \frac{\partial T}{\partial r}\right)+ \\
& +\frac{\partial}{r^{2} \partial \varphi}\left(\kappa \frac{\partial T}{\partial \varphi}\right)+\frac{\partial}{\partial z}\left(\kappa \frac{\partial T}{\partial z}\right)+\frac{\partial(\kappa T)}{r \partial r}+Q_{c}+ \\
& +\frac{\mu}{2}\left[\left(\frac{\partial u}{r \partial \varphi}-\frac{\partial v}{\partial r}-\frac{v}{r}\right)^{2}+\left(\frac{\partial v}{\partial z}+\frac{\partial w}{r \partial \varphi}\right)^{2}+\left(\frac{\partial w}{\partial r}+\frac{\partial u}{\partial z}\right)^{2}\right]+
\end{aligned}
$$

Here are: $\rho, \vec{v}=\{u, v, w\}, p, T$ - density, velocity vector, pressure and temperature, respectively, $\mu, \kappa$ dynamic viscosity and heat conductivity, $v=\mu / \rho$ kinematic viscosity coefficient, $c_{V^{-}}$heat capacity, $Q c$ - internal heat generation (e.g. due to cavitation). The water density is constant (incompressible liquid) by comparably small temperature variation, while for the vapour it is a function of time and space (depending on temperature, pressure).

\subsection{Simplification of the Equations}

In the above general form, the partial differential equation (PDE) array (1) is simplified according to the specific flow conditions. A flow in a thin gap between the channels rotating around their axis with a frequency $\omega$, is considered. The centrifugal force due to rotation of the channel is acting by its radius; $r_{0}$ is a radius.

The width of the narrow gap between the channels is small comparing to the radius $r_{0} . \Omega$ is a frequency of the disk's rotation with a channel on it, which creates the centrifugal force acting by the radius $R_{0}$ in the plane of disk's rotation. These forces are projected on the coordinates $r$ and $\varphi$, with account of the distance from the centre of rotation, where the channel is moving with constant speed around the central vertical axis by the tangential trajectories. The centrifugal and Coriolis forces due to rotations are accounted in the equation array (1).

\subsubsection{Stationary inviscid flow}

For the stationary inviscid flow assuming that the flow velocity across the thin gap is small comparing to the two other velocity components, the following rough estimations were got:

$$
\begin{gathered}
\frac{p}{\rho}+\frac{1}{2} w^{2}=\left(\frac{\omega^{3} r_{0} \sin \varphi}{\Omega \cos ^{2} \varphi}-2 \Omega \omega r_{0} \sin \varphi\right) z+ \\
+2 \frac{\omega^{2} r_{0} w}{\Omega \cos \varphi}+C(\varphi), \\
v^{2}=w^{2}+4 \frac{\omega^{2} r_{0} w}{\Omega \cos \varphi}+4 \omega^{2} r_{0}^{2} \ln |\cos \varphi|+ \\
+\Omega^{2} r_{0}\left(2 R_{0}-r_{0} \cos \varphi\right) \cos \varphi+ \\
+2\left(2 \Omega \omega r_{0}-\frac{\omega^{3} r_{0}}{\Omega \cos ^{2} \varphi}\right) z \sin \varphi-2 C(\varphi) .
\end{gathered}
$$

Here $C(\varphi)$ is an arbitrary function calculated from the boundary conditions. The above estimations show specific feature by the upper and bottom points in the channel, where the velocities and pressure have infinity as peculiarity. Also the estimations show $w \approx \sqrt{2} v$. Despite the equations are complex even for the numerical solution, this estimation is valuable for understanding the features in liquid movement inside the gap. It is used for further estimations together with the other models.

The equation array (2) shows that the flow is dramatically oscillating by both coordinates $z, \varphi$. From (2) follows that there are singularity points up and down in a rotating channel, where the pressure 
and velocity have breaks and abrupt growing, which can be damped by viscous forces neglected here.

\subsubsection{Correlation between axial and rotational velocities in rotating channel by $\varphi \approx 0$ :}

From the second equation of the system (2) also approximation follows in a region by $\varphi \approx 0$ :

$$
\begin{gathered}
\frac{p}{\rho}+\frac{1}{2} w^{2}=2 \omega^{2} \frac{r_{0} w}{\Omega}+C(\varphi), \\
v^{2}=w^{2}+4 \omega^{2} \frac{r_{0} w}{\Omega}+\Omega^{2} r_{0}\left(2 R_{0}-r_{0}\right)-2 C(\varphi),
\end{gathered}
$$

where from yields

$$
\begin{gathered}
\frac{p}{\rho}+\frac{1}{2} w^{2}=2 \omega^{2} \frac{r_{0} w}{\Omega}+C(\varphi), \\
v^{2}=2 w^{2}+2 \frac{p}{\rho}+\Omega^{2} r_{0}\left(2 R_{0}-r_{0}\right)-4 C(\varphi) .
\end{gathered}
$$

The first equation shows that dynamic pressure is proportional to the flow velocity along the axis (inflow to the channel in particular), the radius of a channel and the ratio $\omega^{2} / \Omega . C(\varphi)=0$ in case of symmetrical distribution by the angle.

The higher is rotation in the channel, the higher is pressure, while the main rotation decrease the pressure (here it is acting against the centrifugal force due to rotation in the channel). It is clearly seen that rotational flow is directed from the line $\varphi=0$ up and down - the positive and negative rotational flow velocity $v$. As far as $v^{2} \geq 0$, it yields

$$
w^{2}+4 \omega^{2} \frac{r_{0} w}{\Omega}+\Omega^{2} r_{0}\left(2 R_{0}-r_{0}\right) \geq 0,
$$

which is satisfied by

$$
w \leq-2 \omega^{2} \frac{r_{0}}{\Omega}\left\lfloor 1+\sqrt{1-\frac{\Omega^{4}}{2 \omega^{4}}\left(\frac{R_{0}}{r_{0}}-\frac{1}{2}\right)}\right\rfloor
$$

and

$$
w \geq-2 \omega^{2} \frac{r_{0}}{\Omega}\left\lfloor 1-\sqrt{1-\frac{\Omega^{4}}{2 \omega^{4}}\left(\frac{R_{0}}{r_{0}}-\frac{1}{2}\right)}\right\rfloor .
$$

The first region corresponds to a negative velocity along the axis, which is interesting for use of the reactive force from the channel. The other region covers small negative values and all positive ones.

For the case of small ratio of the rotation speeds, $\Omega^{4} / \omega^{4}<<1$, satisfying the condition

$$
\frac{\Omega^{4}}{2 \omega^{4}}\left(\frac{R_{0}}{r_{0}}-\frac{1}{2}\right)<<1
$$

because all other values are of the same order, the above conditions are simplified in a linear approach:

$$
\begin{gathered}
w \leq-2 \omega^{2} \frac{r_{0}}{\Omega}\left[2-\frac{\Omega^{4}}{4 \omega^{4}}\left(\frac{R_{0}}{r_{0}}-\frac{1}{2}\right)\right]= \\
-4 \omega^{2} \frac{r_{0}}{\Omega}+\frac{\Omega^{3}}{2 \omega^{2}}\left(R_{0}-\frac{r_{0}}{2}\right), w \geq-\frac{\Omega^{3}}{2 \omega^{2}}\left(R_{0}-\frac{r_{0}}{2}\right) .
\end{gathered}
$$

We have applied the Taylor series expansion up to the linear terms by a small value above.

If both rotations are counter clockwise and velocity along the axis in the channel is negative so that tangential line to the main rotation circle is directed the same as the rotation velocity, then negative $w$ means that flow at the exit from the channel is creating the reactive force to the main rotation.

The real values of the velocity are in case of

$$
\frac{\Omega^{4}}{2 \omega^{4}}\left(\frac{R_{0}}{r_{0}}-\frac{1}{2}\right) \leq 1, \frac{R_{0}}{r_{0}} \leq \frac{2 \omega^{4}}{\Omega^{4}}+\frac{1}{2} .
$$

\subsubsection{Correlation between axial and rotational velocities in rotating channel at the $\varphi \approx \pm \pi$ :}

On the opposite side $(\varphi \approx \pm \pi)$ we have

$$
\begin{gathered}
\frac{p}{\rho}+\frac{1}{2} w^{2}=-2 \omega^{2} \frac{r_{0} w}{\Omega}+C(\varphi), \\
v^{2}=w^{2}-4 \omega^{2} \frac{r_{0} w}{\Omega}-\Omega^{2} r_{0}\left(2 R_{0}+r_{0}\right)-2 C(\varphi),
\end{gathered}
$$

where from the dynamic pressure is negative (like the water from the right side is sucked to the left side of the rotating channel). The rotation velocity is small. For example, by $\mathrm{C}=0$ it is estimated as

$$
v^{2}=w^{2}-4 \omega^{2} \frac{r_{0} w}{\Omega}-\Omega^{2} r_{0}\left(2 R_{0}+r_{0}\right) \geq 0 .
$$

This condition is satisfied by

$$
w \leq 2 \omega^{2} \frac{r_{0}}{\Omega}\left[1-\sqrt{1+\frac{\Omega^{4}}{2 \omega^{4}}\left(\frac{R_{0}}{r_{0}}+\frac{1}{2}\right)}\right\rfloor<0,
$$

and

$$
w \geq 2 \omega^{2} \frac{r_{0}}{\Omega}\left\lfloor 1+\sqrt{1+\frac{\Omega^{4}}{2 \omega^{4}}\left(\frac{R_{0}}{r_{0}}+\frac{1}{2}\right)}\right\rfloor>0 .
$$

For the case of small ratio of the rotation speeds, $\Omega^{4} / \omega^{4}<<1$, when the condition is satisfied

$$
\frac{\Omega^{4}}{2 \omega^{4}}\left(\frac{R_{0}}{r_{0}}+\frac{1}{2}\right)<<1
$$

because all other values are of the same order, the above conditions are simplified in a linear approach: 


$$
\begin{gathered}
w \leq-\frac{\Omega^{3}}{2 \omega^{2}}\left(R_{0}+\frac{r_{0}}{2}\right), \\
w \geq 2 \omega^{2} \frac{r_{0}}{\Omega}\left[2+\frac{\Omega^{4}}{4 \omega^{4}}\left(\frac{R_{0}}{r_{0}}+\frac{1}{2}\right)\right] .
\end{gathered}
$$

The second case does not correspond to the need of using reactive force of the exit flow from the channel in pushing the counter clockwise main rotation.

\subsection{Estimation of the Stationary Flow of Incompressible Liquid}

For the flow in a narrow gap we assume that a flow velocity $u$ in the cross sectional direction can be neglected. Then we have only the rotational and longitudinal components of the velocity $(v, w)$ in the gap of the channels. The flow gradient across the thin gap is accounted $(\partial u / \partial r \neq 0)$ because despite the low velocity it may be remarkable due to small width of the gap. Thus, from (1) follows:

$$
\begin{gathered}
\frac{\partial u}{\partial r}+\frac{u}{r}+\frac{\partial v}{r \partial \varphi}+\frac{\partial w}{\partial z}=0 \\
u \frac{\partial u}{\partial r}+\frac{v \partial u}{r \partial \varphi}+w \frac{\partial u}{\partial z}-\frac{v^{2}}{r}=v\left(\frac{\partial^{2} u}{\partial r^{2}}+\frac{1}{r} \frac{\partial u}{\partial r}-\frac{2}{r^{2}} \frac{\partial v}{\partial \varphi}\right)+ \\
+\left(R_{0}-r_{0} \cos \varphi\right) \Omega^{2} \cos \varphi-r_{0} \omega^{2}+2(w \Omega \cos \varphi-v \omega), \\
\frac{v \partial v}{r \partial \varphi}+\frac{w \partial v}{\partial z}+\frac{1}{r \rho} \frac{\partial p}{\partial \varphi}=v\left(\frac{\partial^{2} v}{\partial r^{2}}+\frac{1}{r^{2}} \frac{\partial^{2} v}{\partial \varphi^{2}}+\frac{\partial^{2} v}{\partial z^{2}}+\right. \\
\left.+\frac{1}{r} \frac{\partial v}{\partial r}-\frac{v}{r^{2}}\right)+2(u \omega-w \Omega \sin \varphi)+ \\
+\left(r_{0} \cos \varphi-R_{0}\right) \Omega^{2} \sin \varphi \\
\frac{v \partial w}{r \partial \varphi}+w \frac{\partial w}{\partial z}=-\frac{1}{\rho} \frac{\partial p}{\partial z}+v\left(\frac{\partial^{2} w}{\partial r^{2}}+\frac{1}{r^{2}} \frac{\partial^{2} w}{\partial \varphi^{2}}+\right. \\
\left.+\frac{1}{r} \frac{\partial w}{\partial r}+\frac{\partial^{2} w}{\partial z^{2}}\right)+2 \Omega(u \cos \varphi-v \sin \varphi) .
\end{gathered}
$$

The liquid is incompressible and the pressure gradient across the gap is supposed negligible due to the thickness of the gap, $\partial p / \partial r=0$.

\subsubsection{The Isothermal Flow}

The isothermal flow is analyzed further for simplicity; therefore, the energy equation is omitted in the equation array (3).

Due to a small width of the gap it is assumed that the coordinate across the gap is nearly constant $\left(r=r_{0}\right.$ the centre line of the gap), and $\partial / \partial \varphi \approx 0$ - stable rotational flow inside the channel with a constant rotation speed $(\partial v / \partial \varphi=0, \partial p / \partial \varphi=0)$. Then the gradients of the cross flow velocity $\partial u / \partial \varphi, \partial u / \partial z$ are small because $u$ is small and the characteristic ranges of the rotational and longitudinal (along the axis of the channel) coordinates are much higher than the cross sectional distance in a gap.

The above stated allows simplifying the array (3):

$$
\begin{gathered}
v^{2}=r_{0}^{2} \omega^{2}-v r_{0}\left(\frac{\partial^{2} u}{\partial r^{2}}+\frac{1}{r_{0}} \frac{\partial u}{\partial r}\right)-2 r_{0}(w \Omega \cos \varphi-v \omega)+ \\
-\left(R_{0}-r_{0} \cos \varphi\right) \Omega^{2} r_{0} \cos \varphi \\
\frac{v \partial w}{r_{0} \partial \varphi}+w \frac{\partial w}{\partial z}=v\left(\frac{\partial^{2} w}{\partial r^{2}}+\frac{1}{r} \frac{\partial w}{\partial r}+\frac{\partial^{2} w}{\partial z^{2}}\right)+ \\
+2 \Omega(u \cos \varphi-v \sin \varphi)-\frac{1}{\rho} \frac{\partial p}{\partial z}, \frac{\partial u}{\partial r}+\frac{\partial w}{\partial z}=0 \\
\frac{v \partial v}{r_{0} \partial \varphi}+\frac{w \partial v}{\partial z}=v\left(\frac{\partial^{2} v}{\partial r^{2}}+\frac{\partial^{2} v}{\partial z^{2}}+\frac{1}{r_{0}} \frac{\partial v}{\partial r}-\frac{v}{r^{2}}\right)+ \\
+\left(r_{0} \cos \varphi-R_{0}\right) \Omega^{2} \sin \varphi+2(u \omega-w \Omega \sin \varphi) .
\end{gathered}
$$

If velocity across the gap is totally neglected but the rotational velocity has non-zero gradient, e.g. $\partial v / \partial \varphi \neq 0$, then from the equation array (3) yields

$$
\begin{gathered}
\frac{1}{r_{0}} \frac{\partial v}{\partial \varphi}+\frac{\partial w}{\partial z}=0, \quad v^{2}=v \frac{2}{r_{0}} \frac{\partial v}{\partial \varphi}+r_{0}^{2} \omega^{2}+ \\
-r_{0}\left(R_{0}-r_{0} \cos \varphi\right) \Omega^{2} \cos \varphi-2 r_{0}(w \Omega \cos \varphi-v \omega) \\
\frac{v \partial w}{r_{0} \partial \varphi}+w \frac{\partial w}{\partial z}=-\frac{1}{\rho} \frac{\partial p}{\partial z}+2 \Omega(u \cos \varphi-v \sin \varphi)+ \\
+v\left(\frac{\partial^{2} w}{\partial r^{2}}+\frac{1}{r_{0}} \frac{\partial w}{\partial r}+\frac{1}{r_{0}^{2}} \frac{\partial^{2} w}{\partial \varphi^{2}}+\frac{\partial^{2} w}{\partial z^{2}}\right) \\
\frac{v \partial v}{r_{0} \partial \varphi}+\frac{w \partial v}{\partial z}+\frac{1}{\rho r_{0}} \frac{\partial p}{\partial \varphi}= \\
+\left(\frac{\partial^{2} v}{\partial r^{2}}+\frac{1}{r_{0}^{2}} \frac{\partial^{2} v}{\partial \varphi^{2}}+\frac{\partial^{2} v}{\partial z^{2}}+\frac{1}{r_{0}} \frac{\partial v}{\partial r}-\frac{v}{r_{0}^{2}}\right)+ \\
+\left(r_{0} \cos \varphi-R_{0}\right) \Omega^{2} \sin \varphi+2(u \omega-w \Omega \sin \varphi)
\end{gathered}
$$

where $\partial p / \partial \varphi \neq 0$ in case of uniform rotation. In a stationary case, when the velocity component $v$ is due to the rotation and constant by $\varphi$, then from the first equation (5) follows $\partial w / \partial z=0$. And the second equation (5) determines a flow velocity $v$ due to rotation.

\subsubsection{The Inviscid Approximation}

For inviscid approximation, (5) is more simplified: 


$$
\begin{gathered}
\frac{1}{r_{0}} \frac{\partial v}{\partial \varphi}+\frac{\partial w}{\partial z}=0, v^{2}=2 r_{0}(v \omega-w \Omega \cos \varphi)+ \\
+r_{0}^{2} \omega^{2}-r_{0}\left(R_{0}-r_{0} \cos \varphi\right) \Omega^{2} \cos \varphi \\
\frac{v \partial w}{r_{0} \partial \varphi}+w \frac{\partial w}{\partial z}=-\frac{1}{\rho} \frac{\partial p}{\partial z}+2 \Omega(u \cos \varphi-v \sin \varphi) \\
\frac{v \partial v}{r_{0} \partial \varphi}+\frac{w \partial v}{\partial z}+\frac{1}{\rho r_{0}} \frac{\partial p}{\partial \varphi}=2(u \omega-w \Omega \sin \varphi)+ \\
+\left(r_{0} \cos \varphi-R_{0}\right) \Omega^{2} \sin \varphi
\end{gathered}
$$

where from the following robust estimation is got

$$
\begin{aligned}
& \frac{1}{r_{0}} \frac{\partial v}{\partial \varphi}=-\frac{\partial w}{\partial z}, v^{2}=2(v \omega-w \Omega \cos \varphi) r_{0}+ \\
& +r_{0}^{2} \omega^{2}+\left(r_{0} \cos \varphi-R_{0}\right) \Omega^{2} r_{0} \cos \varphi
\end{aligned}
$$

or, with account of $v \approx \omega r_{0}$, in a first approach by governing rotational flow, (7) yields the following:

$$
\begin{aligned}
& w \approx \frac{\Omega^{2}\left(R_{0}-r_{0} \cos \varphi\right) \cos \varphi-4 \omega^{2} r_{0}}{2 \Omega \cos \varphi}, \\
& \text { by } \varphi=0: w \approx \frac{\Omega\left(R_{0}-r_{0}\right)}{2}-2 \frac{\omega^{2} r_{0}}{\Omega},
\end{aligned}
$$

where from follows that by two rotations, the axial velocity is determined by the angle of rotation and the ratio of radiuses and rotation speeds.

For example, by $\varphi=0$ there are the next limit cases: $R_{0}>>r_{0}, w \approx 0.5 \Omega R_{0}-2 \omega^{2} r_{0} / \Omega$, so that by $\Omega>>\omega: \quad w \approx \Omega R_{0} / 2$ because $\Omega R_{0}>\omega r_{0}$ and $\omega / \Omega<<1$. By $\omega>>\Omega$ : $w \approx-2 \omega^{2} r_{0} / \Omega$ due to $\Omega R_{0}<<\omega r_{0}, \omega / \Omega>>1$. This has a reasonable physical explanation: with a higher speed of the channel's rotation, the axial flow in a channel is substantially higher than the rotational one.

Thus, by the main rotation overwhelming by a speed the channel's rotation, the flow along the axis is going with a half of the tangential velocity caused due to the main rotation. But in the opposite case, when channel is rotating faster than the disk, the tangential velocities at the edge of channel and at the edge of the disk can be of the same order. Then the axial velocity in a channel is much higher than the tangential one. And it can be negative or positive depending on the directions of the rotations.

The task is considered in cylindrical coordinate system attached to the tangential line of the disk. For example, $w \approx 0.5\left(R_{0}-r_{0} \cos \varphi\right)-20 v / \cos \varphi$ is got by $\omega / \Omega=10$, so that much higher flow velocity is along the axis comparing to the rotation in a channel, with the picks up and down in gap, where $\cos \varphi=0$.
Despite the centrifugal and Coriolis forces caused by two different rotations act in variable by angle directions, these points of dramatic grow and changes of the directions $(\varphi=\pi / 2+\pi n, n=1,2, \ldots)$ look something strange. Therefore, we consider the above estimations as the very approximate ones.

\subsubsection{Viscous flow in the narrow gap}

Also it is interesting to consider the viscous flow due to the small gap (high viscous and capillary forces) and high viscosity (like the conditions in the Tesla turbine for example). Neglecting the convective terms and derivatives by $r$ yields from (5) the following:

$$
\begin{gathered}
\frac{1}{r_{0}} \frac{\partial v}{\partial \varphi}+\frac{\partial w}{\partial z}=0, \quad v^{2}=v \frac{2}{r_{0}} \frac{\partial v}{\partial \varphi}+r_{0}^{2} \omega^{2}+ \\
-r_{0}\left(R_{0}-r_{0} \cos \varphi\right) \Omega^{2} \cos \varphi-2 r_{0}(w \Omega \cos \varphi-v \omega) \\
\frac{1}{\rho} \frac{\partial p}{\partial z}=2 \Omega(u \cos \varphi-v \sin \varphi)+v\left(\frac{1}{r_{0}^{2}} \frac{\partial^{2} w}{\partial \varphi^{2}}+\frac{\partial^{2} w}{\partial z^{2}}\right) \\
\frac{1}{\rho r_{0}} \frac{\partial p}{\partial \varphi}=v\left(\frac{1}{r_{0}^{2}} \frac{\partial^{2} v}{\partial \varphi^{2}}+\frac{\partial^{2} v}{\partial z^{2}}-\frac{v}{r_{0}^{2}}\right)+ \\
+\left(r_{0} \cos \varphi-R_{0}\right) \Omega^{2} \sin \varphi+2(u \omega-w \Omega \sin \varphi) .
\end{gathered}
$$

Numerical solution of the equation array (8) with the boundary conditions (all values in SI, $\Omega=50$, $\omega=150 r_{0}=0.06, R_{0}=0.18$, liquid - water):

$$
p=10^{5}, u=0, v=3, w=-0.5,
$$

is presented in Figs 3, 4 for the cross section $z=-1$. The most pulsations of rotational velocity are to the left side of the channel. Pressure is increased in this region up to 20 times, and the most intensive flow along the axis is at $\varphi=\pi$. All parameters of the flow are highly oscillating ( $\varphi$ is assigned as $x$ in figures).

\subsubsection{The test estimation for the rotational flow}

Some more similar estimation was done for the following parameters: $r_{0}=0.05 \mathrm{~m}, \Omega=2400 \mathrm{rpm}=40$ $1 / \mathrm{s}, \omega=24000 \mathrm{rpm}=4001 / \mathrm{s}, \quad w \approx 400 / \cos \varphi+8 \mathrm{~m} / \mathrm{s}$, $v \approx-20 \mathrm{~m} / \mathrm{s} ; \Omega=201 / \mathrm{s}, \omega=1001 / \mathrm{s}, \quad v \approx-5 \mathrm{~m} / \mathrm{s}$, $w \approx 100 / \cos \varphi+4 \mathrm{~m} / \mathrm{s}$. Velocity is growing by $\varphi$ from 0 to $\pi / 2$ but decreasing by $\varphi$ from 0 to $-\pi / 2$ because the resulting centrifugal force is growing from 0 up and down by $\varphi$ counter-currently, flow is going to $\varphi=\pi$ from both sides by the angle.

The peculiarity analysis of the flow in the considered system of 2 rotations is done considering one more problem applying the other approach, to compare the results. Let us perform transformation of 
the equation array (6) with account of the above considered so that to get the following:

$$
\begin{gathered}
\frac{\partial}{\partial \varphi}\left(\frac{p}{\rho}+0.5 v^{2}\right)=2 \omega^{2} r_{0}^{2} \frac{\sin \varphi}{\cos \varphi}+ \\
\quad+r_{0}\left(R_{0}-r_{0} \cos \varphi\right) \Omega^{2} \sin \varphi,
\end{gathered}
$$

and then integrate the equation obtained by $\varphi$ :

$$
\begin{aligned}
\frac{p}{\rho}+0.5 v^{2}= & r_{0} \Omega^{2}\left(0.5 r_{0} \cos ^{2} \varphi-R_{0} \cos \varphi\right)+ \\
& -2 \omega^{2} r_{0}^{2} \ln |\cos \varphi|,
\end{aligned}
$$

where the constant of integration was taken zero by $\varphi=0$. In the obtained expression we have $\ln 0=-\infty$, by $\varphi=\pi / 2=0$, which means that as in the previous analysis it is got one more the same result about the singular points of the pressure at the top and bottom of the channel. This coincides with the previous analysis supporting it ones more. The same with the right and left part of the channel ( $\varphi=0$ and $\varphi=\pi)$.

Total pressure in a flow to the right and to the left of the channel is respectively:
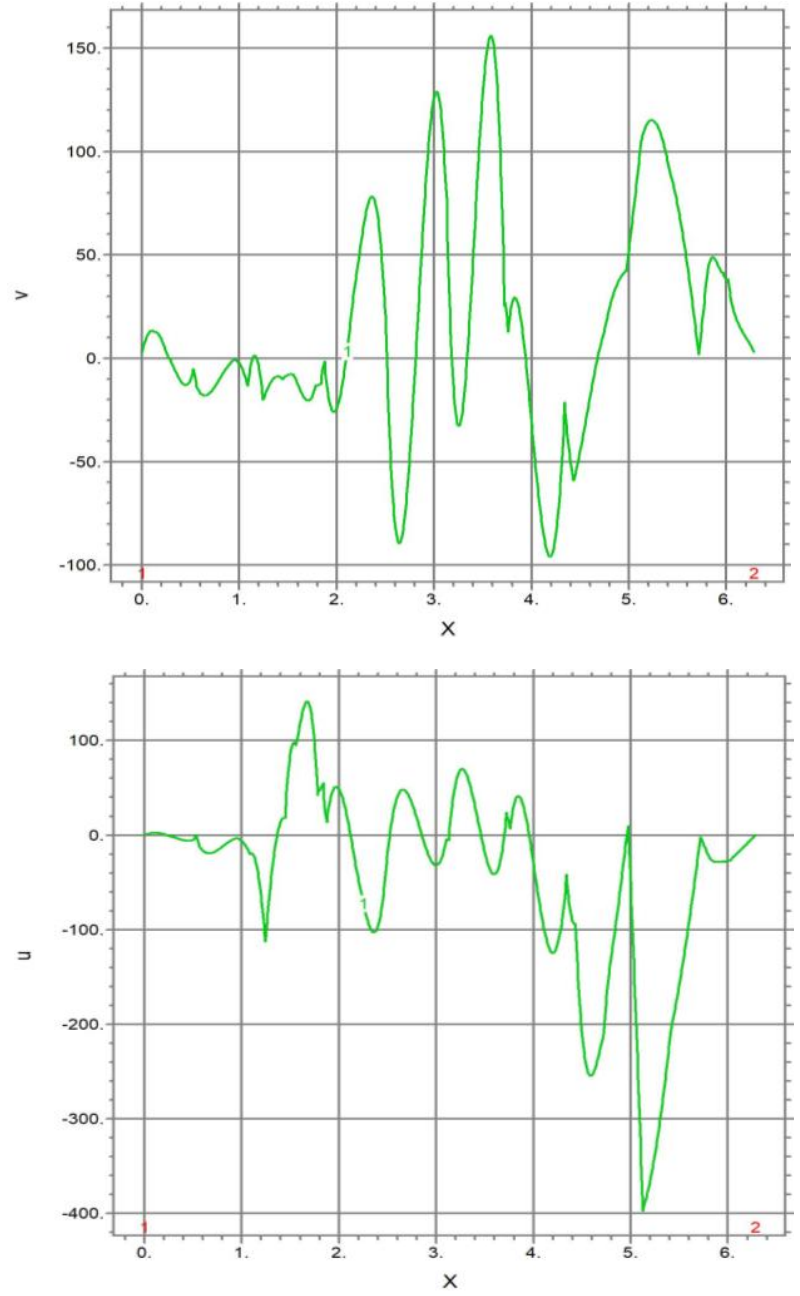

Fig. 3 Rotation fluid velocity $v$ and pulsation velocity $u$ across the gap

$$
\begin{gathered}
p+0.5 \rho v^{2}=\rho r_{0} \Omega^{2}\left(0.5 r_{0}-R_{0}\right), \\
p+0.5 \rho v^{2}=\rho r_{0} \Omega^{2}\left(0.5 r_{0}+R_{0}\right)
\end{gathered}
$$
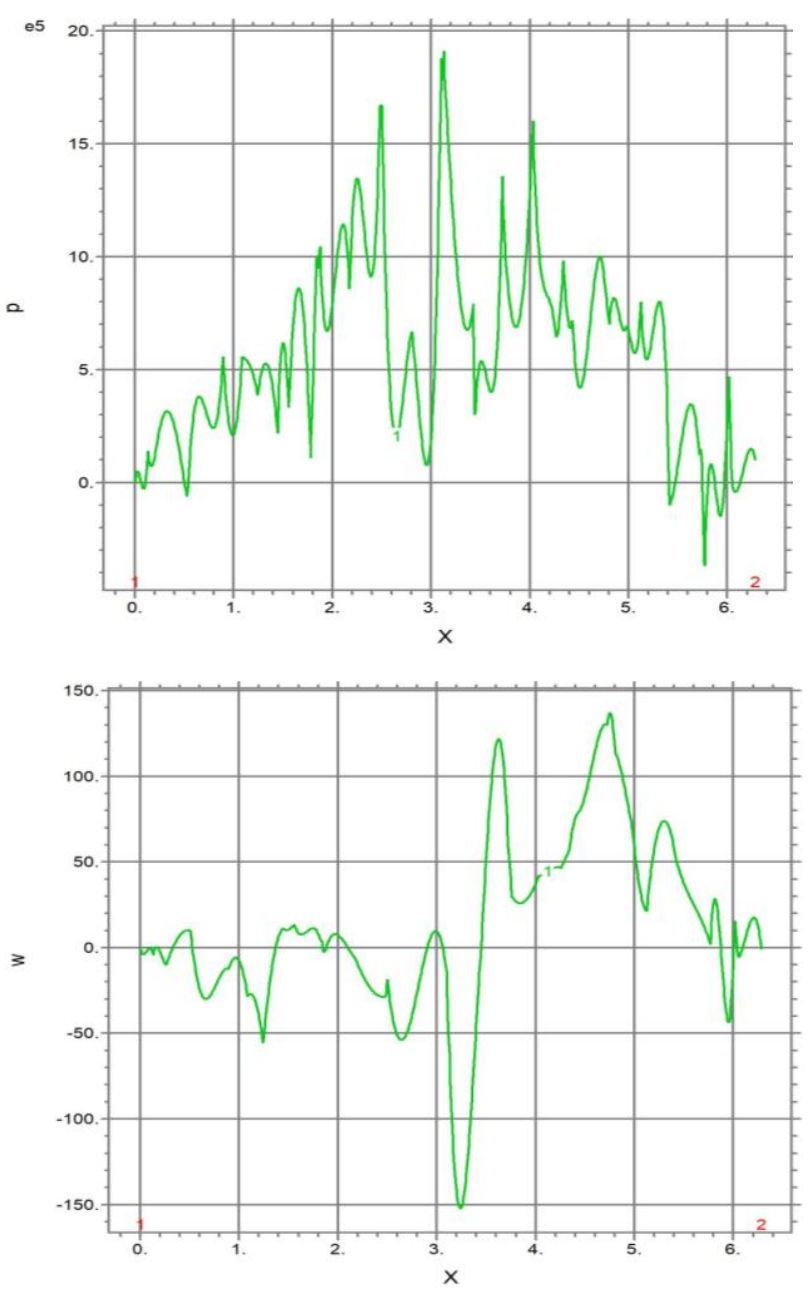

Fig. 4 Flow pressure and velocity $w$ along axis

As shown in (9), to the right it is negative by $R_{0}>r_{0}$, and to the left - positive. This is physically reasonable because the centrifugal forces due to the two rotations (around the main axis and in channel around its axis) are directed opposite to the right, while they are acting in the same direction to the left as shown in Fig. 1 and Fig. 2. The above estimations revealed that the mixing and pressure variation in the channel is highly intensive and it is rapidly changing in the domain depending on the angle of rotation $\varphi$.

The discussed conditions are perfect for the cavitation process inside the volume, which may produce the internal heat generation and other effects. In this simplified model, the curvilinear channels were not considered because they complicate the model dramatically. But as it was shown above, the mixing and pressure variation, even without account of the curvilinear walls, are so high to fit for intensive cavitation processes inside the channel perfectly. 


\section{Flow Stability Analysis}

Let us consider the equation array (1) for the incompressible isothermal flow representing the parameters of the flow as a stable average value plus small deviation in a wavy form:

$$
\begin{gathered}
u=\bar{u}+u^{\prime}, v=\bar{v}+v^{\prime}, w=\bar{w}+w^{\prime}, \\
p=\bar{p}+p^{\prime}, v^{\prime}=V e^{i(k z+m \varphi-\alpha t)}, \\
w^{\prime}=W e^{i(k z+m \varphi-\alpha t)}, p^{\prime}=P e^{i(k z+m \varphi-\alpha t)} .
\end{gathered}
$$

After substitution of the introduced parameters (10) into (1) and linearization of transformation, the equation array similar to (1) is got for the average parameters. The following equations for the perturbations of the parameters qere obtained:

$$
\begin{gathered}
U+i m V+W i k r_{0}=0, \\
i \alpha U-\bar{v} U \frac{i m}{r_{0}}-\bar{w} i k U+2 V \frac{\bar{v}}{r_{0}}=\frac{v}{r_{0}^{2}}(2 V i m+U)+ \\
+2(V \omega-W \Omega \cos \varphi), \\
\frac{i m}{r_{0}} \bar{v} V+\bar{w} i k V+W \frac{d \bar{v}}{d z}+\frac{i m}{\rho r_{0}} P-i \alpha V= \\
=2(U \omega-W \Omega \sin \varphi)-v\left(\frac{m^{2}+1}{r_{0}^{2}} V+k^{2} V\right), \\
\frac{i m}{r_{0}}(\bar{v} W+V \bar{w})+W \frac{d \bar{w}}{d z}+i k W \bar{w}-i \alpha W+\frac{i k}{\rho} P= \\
=2 \Omega(V \sin \varphi-U \cos \varphi)-v\left(\frac{m^{2}}{r_{0}^{2}} W+k^{2} W\right) .
\end{gathered}
$$

\subsection{Estimation of the Flow Peculiarities}

It is clearly observed from the equation array (11) that such wavy oscillations do not satisfy the equations due to dependence on the angle $\varphi$. Therefore, let us estimate the corresponding peculiarities.

If we consider the supplied flow rate $q(1 / \mathrm{s}$, or $\mathrm{kg} / \mathrm{s})$ due to the main rotation with the speed $\Omega(1 / \mathrm{s})$, then we can state that the power introduced by this rotation is approximately $\mathrm{N}=q\left(R_{0} \Omega\right)^{2}(\mathrm{~W})$. Say, it is done by the three channels, the power of each is ideally about $\mathrm{N}_{\mathrm{t}}=\mathrm{N} / 3=q\left(R_{0} \Omega\right)^{2} / 3$.

\subsubsection{Dependence of Two Rotation Speeds}

Without the looses in the system we can estimate the power of the channels by their rotation of the one third of total flow rate, $\mathrm{N}_{\mathrm{t}}=q\left(r_{0} \omega\right)^{2} / 3$. Thus, it follows $q\left(R_{0} \Omega\right)^{2} / 3=q\left(r_{0} \omega\right)^{2} / 3$, where from yields the following approximate correlation:

$$
\omega=\Omega R_{0} / r_{0} .
$$

For $R_{0}=187 \mathrm{~mm}, r_{0}=63 \mathrm{~mm}$ - for the outer channel and 57 for the inner channel. Both channels are of the width $3 \mathrm{~mm}$. It is distributed flow by the rotation and movement along the axes in the channel. Rotational flow is the most. Let us take for the estimation $r_{0}=60 \mathrm{~mm}$, then it results from (12): $\omega=3.1 \Omega$. With account of the losses it may be taken approximately $\omega \approx 3 \Omega$.

\subsubsection{Equations for Amplitude Perturbations} Now from the equations (11) follows:

$$
\begin{gathered}
\frac{U}{r_{0}}+\frac{V}{r_{0}} i m+W i k=0, \\
-i \alpha U+\bar{v} U \frac{i m}{r_{0}}+\bar{w} i k U-2 V \frac{\bar{v}}{r_{0}}= \\
2 \Omega(W \cos \varphi-3 V)-\frac{v}{r_{0}^{2}}(2 V i m+U), \quad \\
\frac{i m}{r_{0}}(\bar{v} W+V \bar{w})+W \frac{d \bar{w}}{d z}+i k W \bar{w}-i \alpha W+\frac{i k}{\rho} P= \\
=2 \Omega(V \sin \varphi-U \cos \varphi)-v\left(\frac{m^{2}}{r_{0}^{2}} W+k^{2} W\right), \\
\frac{i m}{r_{0}} \bar{v} V+\bar{w} i k V+W \frac{d \bar{v}}{d z}+\frac{i m}{\rho r_{0}} P-i \alpha V= \\
=2 \Omega(3 U-W \sin \varphi)-v\left(\frac{m^{2}+1}{r_{0}^{2}} V+k^{2} V\right) .
\end{gathered}
$$

The obtained equation array (13) shows that the dependence from the angle of rotation is principal and cannot be neglected. Therefore, it can be used for estimation of the oscillations but not for the precise solution. If the wave numbers $m, k$ are real values, then only harmonic perturbations are available, which are varying in time.

\subsection{Analysis of the Perturbations}

From the equation array (13) yields the following:

$$
\begin{gathered}
U=0, \quad W=-\frac{m}{r_{0} k} V, \quad \alpha=\frac{2 v}{U r_{0}^{2}} m, \\
V\left(\frac{\bar{v}}{r_{0}}+3 \Omega\right)=-\Omega W \cos \varphi,
\end{gathered}
$$




$$
\begin{gathered}
P=\alpha \frac{\rho}{k} W-\frac{\rho}{k} \frac{m}{r_{0}}(\bar{v} W+V \bar{w})-\rho W \bar{w}, \\
W\left[\frac{d \bar{w}}{d z}+v\left(\frac{m^{2}}{r_{0}^{2}}+k^{2}\right)\right]=2 \Omega(V \sin \varphi-U \cos \varphi), \\
P=\frac{\rho r_{0}}{m}\left[\alpha-\left(\frac{m}{r_{0}} \bar{v}+\bar{w} k\right) \mid V,\right. \\
W\left(\frac{d \bar{v}}{d z}+2 \Omega \sin \varphi\right)=6 \Omega U-v\left(\frac{m^{2}+1}{r_{0}^{2}} V+k^{2} V\right) .
\end{gathered}
$$

It is accounted that all equations must satisfy separately for their real and imaginary parts. With account of $U=0$, it can be got $U \approx 0$ from the previous estimations. Then $\alpha=2 v m>U_{0}^{2}$, or $\alpha=\infty$, which means very high frequency of the oscillations without the instability (strong shaking). And further, the equation array (14) is

$$
\begin{gathered}
W=-\frac{m}{r_{0} k} V, m=\left(\frac{\bar{v}}{\Omega}+3 r_{0}\right) \frac{k}{\cos \varphi}, \\
P=\rho V \frac{m}{r_{0} k}\left(\frac{m \bar{v}}{r_{0} k}-\frac{\alpha}{k}\right), \\
k^{2}+2 \sin \varphi \frac{r_{0}}{m v} \Omega k+\frac{1}{v} \frac{d \bar{w}}{d z}+\frac{m^{2}}{r_{0}^{2}}=0, \\
\alpha\left(1+\frac{m^{2}}{r_{0}^{2} k^{2}}\right)=\bar{v} \frac{m^{3}}{r_{0}^{3} k^{2}}+\frac{m}{r_{0}} \bar{v}+\bar{w} k \\
\frac{m}{r_{0} k}\left(\frac{d \bar{v}}{d z}+2 \Omega \sin \varphi\right)=v\left(\frac{m^{2}+1}{r_{0}^{2}}+k^{2}\right) .
\end{gathered}
$$

The two contradictions to the assumptions made follow from the (15): dependence of the perturbations on the angle $\varphi$ and impossibility to satisfy the equations 3 and 5 due to $\alpha=\infty$. Therefore, let us consider perturbations $e^{i(k z+m \varphi-\alpha t)}$ with $k=k_{r}+i k_{i}$, $\alpha=\alpha_{r}+i \alpha_{i}$, where $k_{r}, k_{i}$ are the real and imaginary parts of the wave number $k$, similarly $-\alpha_{r}, \alpha_{i}$. Then the perturbations have the following form: $e^{i(k z+m \varphi-\alpha t)}=e^{i\left(k_{r} z+m \varphi-\alpha_{r} t\right)} e^{-k_{i} z+\alpha_{i} t}, \quad$ so that the perturbations may be wavy by $z, \varphi$ and time, and exponentially growing or decreasing by the axis, while the law by time must be got from solution of the equations. Thus, from (15) yields $U=k_{i} r_{0} W$ and:

$$
\alpha_{r}=\frac{2 v}{U r_{0}^{2}} m V-m \frac{\bar{v}}{r_{0}}-\bar{w} k_{r}, W=-\frac{m}{k_{r} r_{0}} V,
$$

$$
\begin{gathered}
\alpha_{i}=\frac{2 \Omega}{U}(W \cos \varphi-3 V)-\frac{v}{r_{0}^{2}}-2 V \frac{\bar{v}}{U r_{0}}-\bar{w} k_{i}, \\
\frac{i m}{r_{0}} \bar{v} V+\bar{w}\left(i k_{r}-k_{i}\right) V+W \frac{d \bar{v}}{d z}+\left(\alpha_{i}-i \alpha_{r}\right) V= \\
=-v\left[\frac{m^{2}+1}{r_{0}^{2}} V+\left(k_{r}^{2}-k_{i}^{2}+2 i k_{r} k_{i}\right) V\right]+ \\
+2 \Omega(3 U-W \sin \varphi)-\frac{i m}{\rho r_{0}} P, \\
\frac{i m}{r_{0}}(\bar{v} W+V \bar{w})+W \frac{d \bar{w}}{d z}+\left(i k_{r}-k_{i}\right) W \bar{w}+ \\
+\left(\alpha_{i}-i \alpha_{r}\right) W=2 \Omega(V \sin \varphi-U \cos \varphi)+ \\
+\frac{k_{i}-i k_{r}}{\rho} P-v\left[\frac{m^{2}}{r_{0}^{2}} W+\left(k_{r}^{2}-k_{i}^{2}+2 i k_{r} k_{i}\right) W\right] .
\end{gathered}
$$

Here $\alpha_{r}$ determines the oscillation frequency by time, while $\alpha_{i}$ is responsible for growing of the oscillations $\left(\alpha_{i}>0\right)$ or their decreasing $\left(\alpha_{i}<0\right)$ in time. The first four correlations for the $\alpha_{r}, \alpha_{i}$ and $U, W$ may be transformed from (16) as follows:

$$
\begin{gathered}
\alpha_{r}=-\frac{2 v k_{r}}{k_{i} r_{0}^{2}}-m \frac{\bar{v}}{r_{0}}-\bar{w} k_{r}, \\
U=-k_{i} \frac{m}{k_{r}} V, W=-\frac{m}{k_{r} r_{0}} V, \\
\alpha_{i}=\frac{2 \Omega}{k_{i}}\left(\frac{\cos \varphi}{r_{0}}+3 \frac{k_{r}}{m}\right)-\frac{v}{r_{0}^{2}}+2 \frac{\bar{v} k_{r}}{k_{i} m r_{0}}-\bar{w} k_{i} .
\end{gathered}
$$

Detail study of the (16), (17) is the separate subject.

\section{Conclusion}

The modeling revealed interesting features of the rotational flow due to the simultaneous rotations in two perpendicular directions. The flow equations with account of the two centrifugal and Coriolis forces were analyzed. Complex flow in a gap of the channel located on the disk rotating around the vertical axis was considered at first.

The conditions created in a flow by alternating volumetric mass forces can fit for the intensive stretching of a liquid, cavitation and other interesting phenomena. The revealed unique processes will be studied more in deep, both theoretically as well as experimentally as far as they may have strong applications. 


\section{References:}

[1] Pascal Belleville, Lhadi Nouri and Jack Legrand, Mixing Characteristics in the Torus Reactor, Chem. Eng. Technol., 1992, No.15, pp. 282289.

[2] Wereley S.T. \& Lueptow R.M., Inertial particle motion in a Taylor Couette rotating filter, Phys. Fluids, 11, 1999, pp. 325.

[3] Smits A., Auvity B. \& Sinha M., Taylor-Couette flow with a shaped inner cylinder, Bull. Am. Phys. Soc., 45(9), 2000, pp. 37.

[4] Andersen A., Bohr T., Stenum B., Rasmussen J.J., Lautrup B. Anatomy of bathtub vortex, Phys. Rev. Lett., Vol.91, 2003, pp. 104502-1-4.

[5] https://www.rt.com/usa/269614-vortex-damlake-texoma/

[6] Hsieh J. S. Engineering Thermodynamics. Prentice-Hall, 1993.

[7] Torricelli's experiment. Simple barometer. PhysicMax. Retrieved 7 December 2016.

[8] Pascal// Encyclopedia of Physics/ Ed. A.M. Prokhorov. M.: Great Russian Encyclopedia, 1992, v. 3: Magnetoplasma - Poynting's theorem, pp. 549-550.

[9] Veritasium. The Most Amazing Thing About Trees. https://www.stem.org.uk/resources/ elibrary/resource/424975.

[10] Simcha Srebnik and Abraham Marmur. Negative Pressure within a Liquid-Fluid Interface Determines Its Thickness, Langmuir, vol. 36 (27), 7943-7947, 2020.

[11] Attila R., Katalin Martinás, L.P.N Rebelo. Thermodynamics of Negative Pressures in Liquids, Journal of Non-Equilibrium Thermodynamics, January, vol. 23, No 4, 351375, 1998.

[12] Kauzmann W., Kinetic theory of gases, W.A. Benjamin Inc., NY, 1996.

[13] Hess S. Rheology and shear induced structures in fluids. In: Lecture notes in physics 381, Rheological modelling: Thermodynamical and statistical approaches. Eds.: Casas-Vasquez J., Jou D., p. 51, Springer-Verlag, BerlinHeidelberg, 1991.

[14] Kell G.S. Early observations of negative pressures in liquids. Am. J. Phys., 51, 1038, 1983.

[15] Zheng Q., Durben D.J., Wolf G.H., Angel C.A., Liquids at large negative pressures: water at the homogeneous nucleation limit, Science, 254, 829, 1991.

[16] Trevena D.H. Cavitation and tension in liquids, Adam Hilger, Bristol, 1987.
[17] Temperley H.N.V., Chambers L.L.G. The behaviour of water under hydrostatic tension: I, Proc. Roy. Soc., 58, 420, 1946.

[18] Temperley H.N.V. The behaviour of water under hydrostatic tension: II, Proc. Roy. Soc., 58, 436, 1946.

[19] Temperley H.N.V. The behaviour of water under hydrostatic tension: III, Proc. Roy. Soc., 59, 199, 1947.

[20] Briggs L.J. Limiting negative pressure of water. J. Appl. Phys., 21, 721, 1950.

[21] Hayward A.T.J. Measuring the extensibility of liquids, Nature, 202, 481, 1964.

[22] Apfel R.E. The tensile strength of liquids. Sci. Amer., 227, 581, 1972.

[23] Henderson S.J., Speedy R.J. A BerthelotBourdon tube method for studying water under tension. J. Phys. E. Sci. Instrum., 13, 778, 1980.

[24] Henderson S.J., Speedy R.J. Temperature of maximum density in water at negative pressure. J. Phys. Chem., 91, 3062, 1987.

[25] Henderson S.J., Speedy R.J. Melting temperature of ice at positive and negative pressure. J. Phys. Chem., 91, 3069, 1987.

[26] Green J.L., Durben D.J., Wolf G.H. Angell C.A. Water and solutions at negative pressure: Raman spectroscopy study to -80 megapascals. Science, 249, 649, 1990.

[27] Vinogradov V.E., Sinitsin E.N., Kotelnikov N.A. Effect of Subcooling on the Reaction Force in Discharge of Flashing Water. Fluid Mechanics-Soviet Research,1987, vol. 16, No. 5, p. 39-41.

[28] Vinogradov V.E., Gissatulina S.V., Sinitsyn YE.N.Maximum Tensile Forces in n-Pentane. Fluid Mechanics Research, 1992, vol.21, No.4, p. 50-54.

[29] Vinogradov V.E., Pavlov P.A. Liquid Boilingup at negative pressures. Proceeding the International Symposium on the Physics of Heat Transfer in Boiling and Condensation. Moscow, 1997, p. 57-60.

[30] Vinogradov V.E., Pavlov P.A. Limiting superheat of aqueous solutions at negative pressures. NATO science series: 2: 42 Mathematics, Physics and Chemistry. 2002. Volume 84: Liquids Under Negative Pressure. P.13-22.

[31] Vinogradov V.E., Pavlov P.A. Cavitation strength of water solutions// Journal of Engineering Thermophysics, 2002, Vol. 11, No. 4, pp. 353-363.

\section{Creative Commons Attribution License 4.0 (Attribution 4.0 International, CC BY 4.0)}

This article is published under the terms of the Creative Commons Attribution License 4.0

https://creativecommons.org/licenses/by/4.0/deed.en US 